\title{
Design and Production of Automated Dental Implant Drilling Test System
}

\author{
[Sayedali Mousavi] \\ Department of mechanical engineering, Najafabad Branch, Islamic Azad University, Najafabad, Iran
}

\begin{abstract}
In dental implant surgery, drilling is first step to make hole for insertion of fixture. Different implant system has their sequence and drilling system for making the implant site preparation. To determine drilling system performance in vitro examination under constant condition, it needs accurate experimental apparatus to determine drilling system performances. The use of a novel test system to drill the implant site in preparation for the insertion of the implant was developed in this research.
\end{abstract}

Experimental condition of drilling test such as feed rate, vertical force, RPM, drill forward and backward and temperature should monitor in designed system. The system's software, which consists of robot calibration module, drill plan module, load plan module, drill execution module, and acquisition data module, is programmed based $C \#$ software GUI system for test monitoring. The experimental result showed that all implants were precisely placed at the right location and Positioning accuracy was improved by $90 \%$ ( $p<$ 0.01) in comparison toy hand drilling; and resolution of drilling was improved by $80 \%(p<0.001)$ in comparison to human hand implantation .

\section{Keywords - Drilling, Experimental Test, Implant,Dentalintroduction}

Holding a dental tool for many hours of work is reflected in fatigue and manual tremor, which causes bad handling of rotatory instruments and consequently injuries within the buccal cavity. Surgical robotics is a new technology that holds significant promise. Robotic surgery is often heralded as a new revolution, and it is one of the most talked about subjects in surgery today. The lack of crossover between industrial robotics and medicine, particularly surgery, is at an end. Robotic tele surgical machines have already been used to perform transcontinental cholecystectomy.[1,2] ] It remains to be seen, however, if history will look on the development of the robotic surgery as a profound paradigm shift or as a bump in the road to the something even more important.

Paradigm shift or not, the origin of surgical robotics is rooted in the strengths and weaknesses of its predecessors. Some of the more prominent limitations involve the technical and mechanical nature of the equipment. Inherent in current dental equipment is a loss of haptic feedback (force and tactile).

Sayedali mousavi, Department of mechanical engineering, Najafabad Branch,Islamic Azad University,Najafabad, Iran
Moving the dental drilling instrument is one must move the instrument in the opposite direction from the desired target on the monitor to interact with the site of interest. Current test instruments have restricted degrees of motion; whereas the human wrist and hand have 7 degrees of motion. There is also a decreased sense of touch that makes tissue manipulation more heavily dependent on visualization. The motivation to develop drilling test mechatronics system is rooted in the desire to overcome the limitations of current in-vitro test technologies and to expand the benefits of minimally invasive implant drilling surgery.

The history of robotics in surgery begins with the Puma 560, a robot used to perform neurosurgical biopsies with greater precision. [3,4]. While PROBOT was being developed, Integrated Surgical Supplies developed ROBODOC, a robotic system designed to machine the femur with greater precision in hip replacement surgeries.[5] In the early 1990s, several of the scientists developed a dexterous tele-manipulator for hand surgery. One of their main design goals was to give the surgeon the sense of operating directly on the patient rather than from across the room. While these robots were being developed, general surgeons joined the development team and realized the potential these systems had in ameliorating the limitations of conventional laparoscopic surgery.

Today, many robots and robot enhancements are being researched and developed. Minimally invasive surgeries have developed a master-slave manipulator system that they call ARTEMIS. [5, 6] Several other laboratories are designing and developing systems and models for reality-based haptic feedback in minimally invasive surgery and also combining visual servoing with haptic feedback for robot-assisted surgery.

Another important variable that may influence the biological response to drilled bone is the time which a temperature above a threshold value is obtained. [79].Automated systems for endodontics and polishing were implemented in previous study that could be used by automatic instrument robotic insertion system.[10,11] Up to this point in time, however, the drive to develop and obtain robotic devices has been largely driven by the market. During dental surgery the lack of dental surgery robot is perceptible.

On The other hand, there is no system to help the dentist in the support and handling of the dental equipment such as drilling system in implant surgical procedure. We propose the use of a mechatronic test machine to help and perform to operate automatically for implant dental drilling system. 
Proc. of The Fifth Intl. Conf. On Advances in Mechanical, Aeronautical and Production Techniques - MAPT 2016 Copyright (C) Institute of Research Engineers and Doctors, USA .All rights reserved.

ISBN: 978-1-63248-090-3 doi: 10.15224/ 978-1-63248-090-3-46

\section{Methods}

The advantages of automated test systems overcame many problem of surgical difficulty. They increased dexterity, restore proper hand-eye coordination and an ergonomic position, and improve visualization .In addition by using, this system it made dentist possible to solve. technically difficult or unfeasible works, Dental drilling test system (DDTS) is an agile manipulator robot system for monitoring and testing implant surgical process that developed using 2DOF (degree of freedom) in our project.

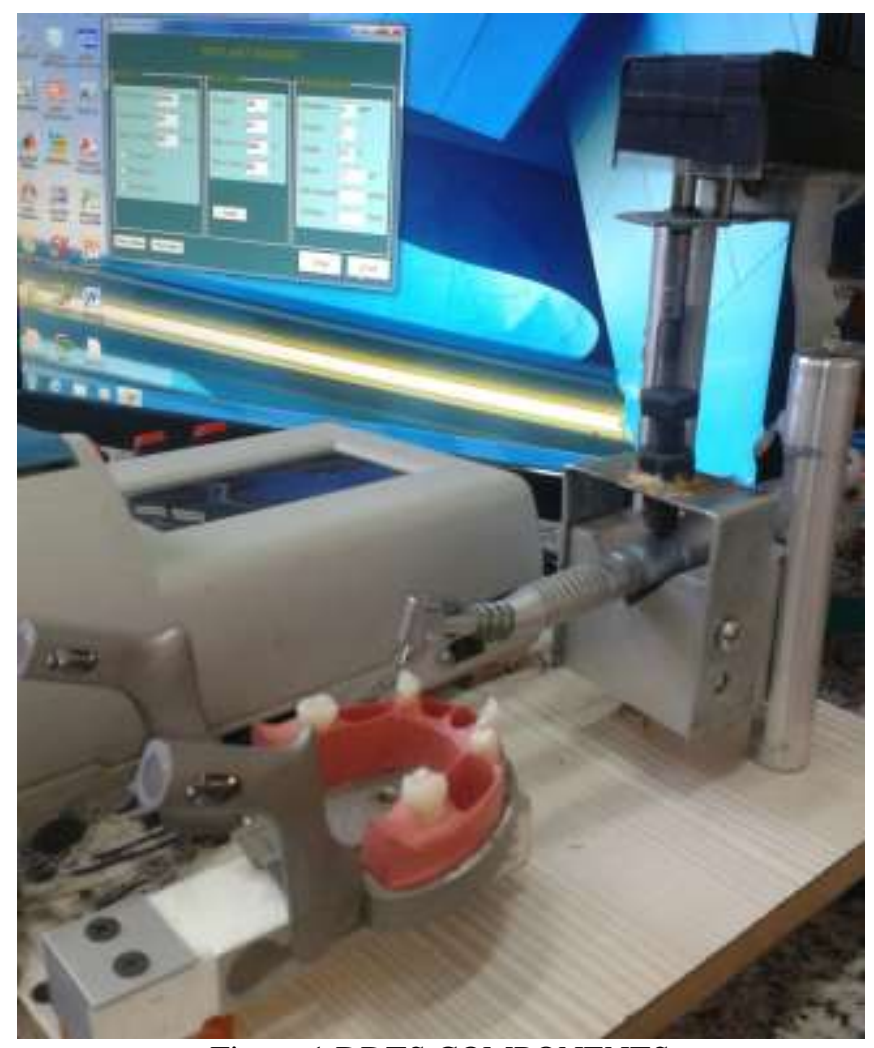

Figure 1.DDTS COMPONENTS

The structure, drilling of hole in jaws and its arrangement algorithm, and motion control and other clinical arrangement system studied DDTS Single manipulator to complete surgical test drilling system was shown in Figure 1, which consisted of the following components: 2DOF, electromechanical gripper, computer, a central feed rate control system with hole -arrangement and force control software for drilling arrangement, motion planning and control, Temperature sensor and irrigation system.

GUI software was programmed based on C\# and BASCOM for graphical user interface module and its operating user friendly for junior dentist. The functions of software were as follows: Choosing or creating implant medical history files of a patient, and insert input parameters of the drilling process such as: feed rate, initial torque, RPM, force, accepted drilling temperature, and adjusts the dental system parameters by dental expert's experience reasonably. (2) Displaying the real time parameter of test as monitoring function on the screen, provide a virtual observation environment for designed checking of drilling system performance. The test system control software was programmed based on BASCOM microcontroller program language. The functions of the control software were used to calibrate drilling initial position for drill position arrangement, creating control data profile for the drill position and feed rate with the control the system performance operation. Experimental procedures thoroughly tested and evaluated before animal testing or human clinical trials on virtual jaw's blocks. The maximum vertical force of this $\mathrm{t}$ system was $200 \mathrm{~N}$, the maximum RPM of motor drilling speed was 250, and the repeated positioning accuracy was $\pm 0.01 \mathrm{~mm}$. In vitro experiments were accomplished for this complete system was implemented by using ITI drilling sequence (Straumann ${ }^{\circledR}$, Sweden) system. The completed drilling for upper as well as lower on jaw of sheep were accomplished with uses of no clinical experience Figure 2 showed the In vitro working process of the single DDTS for complete Drilling process. The stability structure and workspace analysis of DDTS in drilling process were studied. Because of the variable amount of driven motors, was difficult to realize the control and kinematic calculation of this system with kinematic software. Aiming at this problem, for drilling performance strategy was proosed based on the combination of computer decision and dentist analysis with data monitoring system in DDTS as it showed in Figure 2 the monitoring system in DDTS.

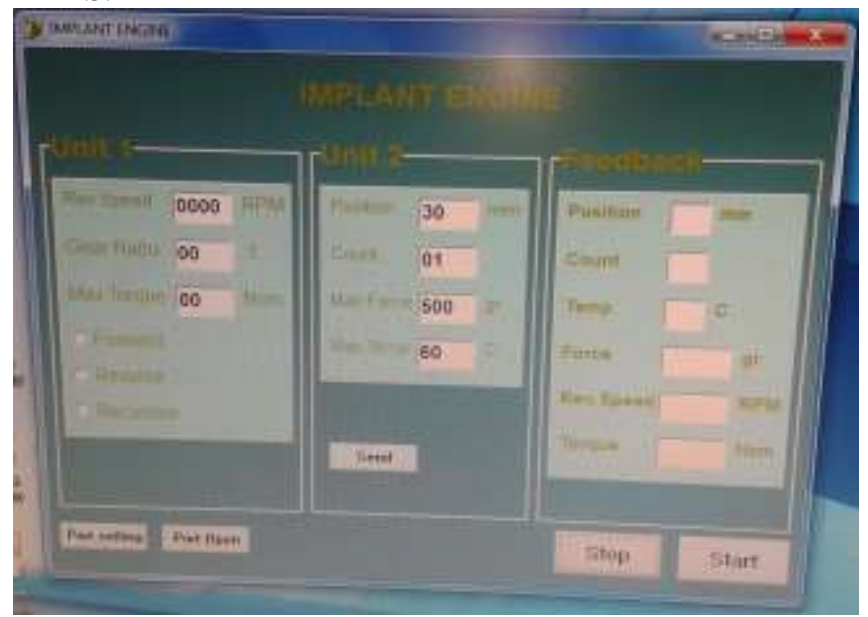

Figure2. The monitoring system in DDTS

DDTS consisted of 2 independent manipulators, a dental feed rate generator, and a rotation mechanism. The DDTS system consisted of an articulated arm with force sensors and actuators that were activated electronically. The mechanism was developed so that the dental hand piece was held simultaneously by the mechanical arm.

The feed rate mechanism was used to achieve the control of the dental drill position, which is used to create a drill position that matches the one from a patient's teeth hole. It could move along its own trail to satisfy the requirement for each tooth's hole placement. This manipulator provided the controls with degrees of freedom (one rotations and one movement) to adjust each tooth placement for its position along Z, lingual, and near-far-medium directions. Two parallel vertical bars were placed under single manipulator and each of the bars was connected to a flexible steel shaft driven by a DC motor. Through coupling, the motor drove the flexible shaft to reciprocate in vertical axis to make drilling system in exact position. This frame moved up and 
down with the rotating shaft of the near-far-medium direction, which moves conjugate with the tooth hole. Two commonly used fixed jaw prosthesis design used to connect an implant and a tooth, a rigid connection, and a non- rigid connection were fabricated and used for experimental verification .Since the drill process helper was fixed on the rotating shaft by a dowelled connection, one traveling freedom was achieved in this way. When the bars rotated inconsistently, the rolling frame rotates; the number of rotation in drilling could be realized. Rotating shaft of direction itself was connected to flexible shaft and driven by a DC motor. It could drive position of drilling system direction .As described above; the DDTS move with two degrees of freedom .The mechanical design in different view was shown in figure3. Thus, the amount of displacement that drove motors was decreased to $5 \mathrm{~cm}$.

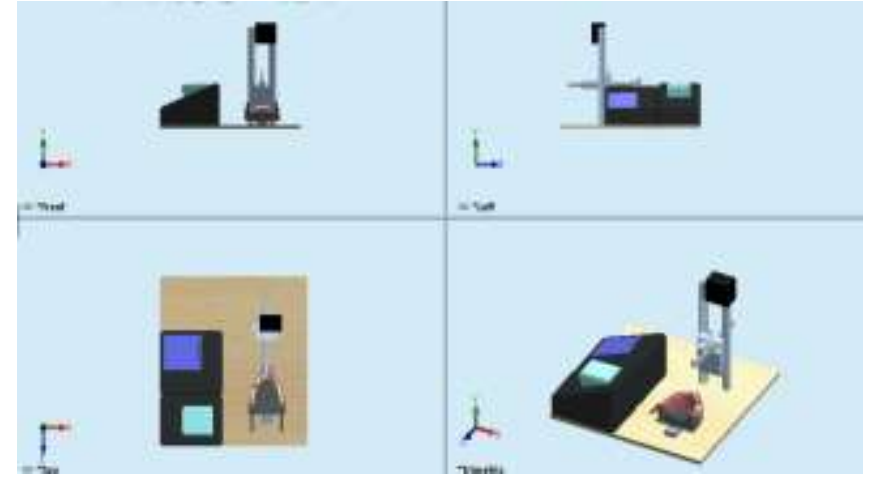

Figure 3 .Mechatronic design view

The optimal number of implants and their placement/orientation was studied through the implant force, and the stress/strain analysis of jaw bone tissue with the different drilling posture, but without considering the drilling depth influence on the drill blade force, and the stress/strain analysis of jaw bone tissue. Expert dentists and odontology students were asked to execute tasks to assess positioning accuracy and system resolution. Students performed positioning tasks to evaluate adaptation to the system. The subjects drew the contour of a circle with and without the mechatronic assistant to assess positioning accuracy. Similarly, they made cavities to evaluate resolution and accuracy. Adaptation to the system was evaluated by inserting the drill burr into cavities previously made as it shown in figure 4.

Experimental results were obtained that will be discussed in results section. Furthermore, the mechanism is simple, agile, and easy to control. The related researches about the coordinated control of dental drilling system, high precision synchronous control of 2 DOF multi-manipulator tooth arrangement robot based on software timer, and the kinematic analysis and planning are studied. The manufacturing process of complete denture only takes about 3 minutes using this robot system.

The accuracy of the robotic systems is measured. The repeated Positioning accuracy is $\pm 0.01 \mathrm{~mm}$ for a single manipulator and $\pm 0.10 \mathrm{~mm}$ for the whole DDTS. In Figure 5 the complete hole made by the DDTS system position and resolution accuracy were illustrated.

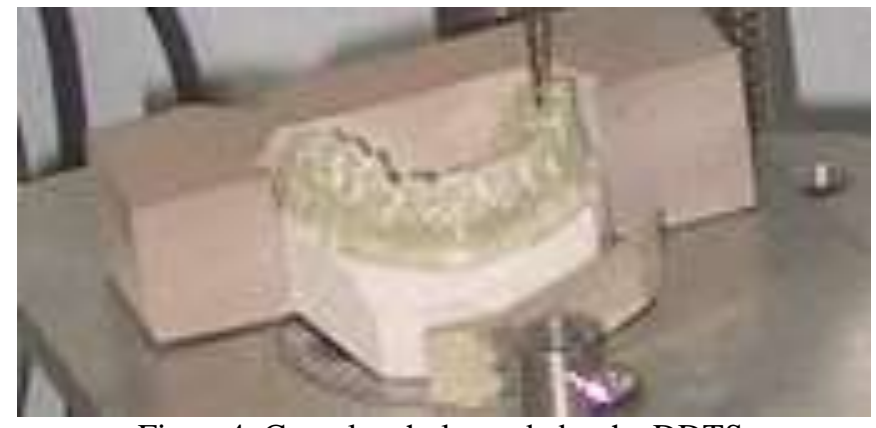

Figure4. Complete hole made by the DDTS

\section{Results}

Several research are currently using dental testing system and publishing data.by implement of these systems, the safety of the patient were insure before operation. The novel system were implemented for these purposes and system experiments showed that the system design was feasible and provided a target registration error of 0.01 $\pm 0.10 \mathrm{~mm}$. The DDTS provided support and stability of dental drilling process the threshold of force required to move the mechanism prevented involuntary movements affecting the quality of work made on cavities. Positioning accuracy was improved by $90 \%(\mathrm{p}<0.01)$ using the system. Similarly, resolution of drilling was improved by $80 \%$ ( $\mathrm{p}$ 0.001 ) in comparison with human hand in vitro examination as it shown in figure 5 .

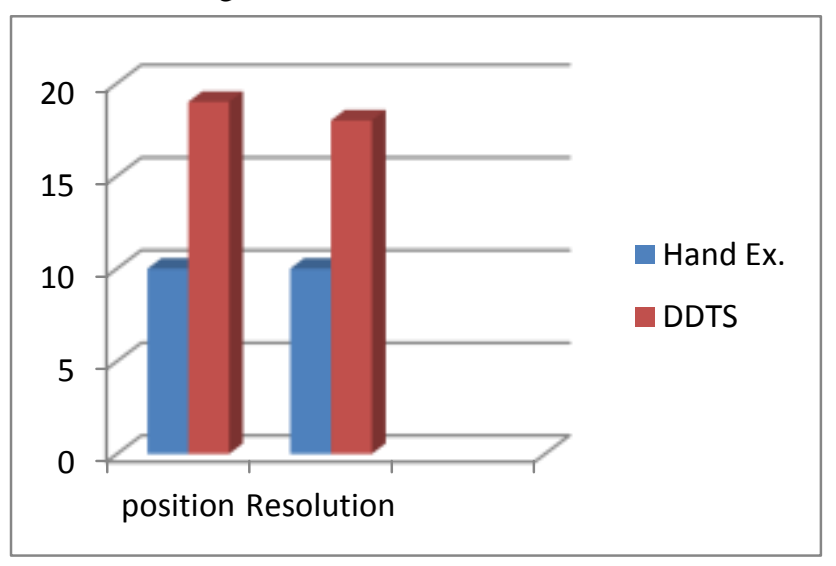

Figure 5. In vitro examination Comparison DDTS and human hand hand-piece drilling for implant hole preparation

\section{Discussion and Conclusion}

To date, mostly studies of feasibility have been conducted, and almost no long-term follow up studies have been performed for medical surgical robot system but the lack of dental surgical robot or machines are perceivable. Many procedures will also have to be redesigned to optimize the use of robotic arms and increase efficiency. However, time will most likely remedy these disadvantages. Research difficulty of dental implantology robot system is 3D reconstruction of preparation oral cavity $\mathrm{CT}$ image, the registration between intraoperative navigation image and preoperative reconstruction image, structure design of robot 
in limited workspace, and trajectory planning of robot. Another disadvantage of these systems is their cost.

In any cases, robotic technology is set to revolutionize surgery by improving procedures, advancing surgical technology, and bringing surgery into the digital age. Furthermore, it has the potential to expand surgical treatment modalities beyond the limitation of human ability. Whether or not the benefit of its usage overcomes the cost to implement it remains to be seen and much remains to be worked out. Although dental robots design feasibility has largely been shown, more prospective randomized trials evaluating efficacy and safety must be undertaken. Further, research must evaluate cost effectiveness or a true benefit over conventional therapy for automatic dental surgery to take full root.

To overcome these problems, we have developed the mechatronic system to assist dentists in handling and testing of the dental drill. This mechanism allows the dentist to manipulate the tool with smooth and precise movements during the preparation of dental cavities with the application of surgical drilling. The mechatronic system minimizes manual risk of human hand during drilling process due to fatigue and reduces the risk of iatrogenic dental injuries position's interactive modification of different loop. For the robot in prosthodontics and orthodontics, it is difficult to detect working condition and realize the operation with multiple obstacles unstructured environment. To facilitate the operation, a kind of intelligent friendly human-computer interaction software should be designed to provide humanization input and feedback for the operators in future researches.

\section{V.References}

[1] Marescaux J, Leroy J, Rubino F, et al. Transcontinental robot-assisted remote telesurgery: feasibility and potential applications. Ann Surg. 2002;235:487-492.

[2] Cheah WK, Lee B, Lenzi JE, et al. Telesurgical laparoscopic cholecystectomy between two countries. Surg Endosc. 2000;14:1085.

[3] Kim VB, Chapman WH, Albrecht RJ, et al. Early experience with telemanipulative robot-assisted laparoscopic cholecystectomy using Da Vinci. Surg Laparosc Endosc Percutan Tech. 2002;12:34-40.

[4] Kwoh YS, Hou J, Jonckheere EA, et al. A robot with improved absolute positioning accuracy for CT guided stereotactic brain surgery. IEEE Trans Biomed Eng. 1988;35:153-161. [PubMed]

[5] Schurr MO, Buess GF, Neisius B, et al. Robotics and telemanipulation technologies for endoscopic surgery: a review of the ARTEMIS project. Advanced robotic telemanipulator for minimally invasive surgery. Surg Endosc. 2000;14:375-381.

[6] Tholey G, Chanthasopeephan T, Hu T, et al. Measuring Grasping and Cutting Forces for Reality-Based Haptic Modeling. Computer Assisted Radiology and Surgery, 17th International Congress and Exhibition, June 2003, London, UK.

[7] Kennedy CW, Desai JP. Force Feedback Using Vision. The 11th International Conference on Advanced Robotics, June 30-July 3, 2003 University of Coimbra, Portugal.

[8] Abouzgia, M. B. and J. M. Symington Effect of drill speed on bone temperature. International Journal of Oral \& Maxillofacial Surgery, 1996, 25(5): 394-399.

[9] Zhang Y.-D., Peng J.-C., and Jiang J.-G., "High precision motion control for multi-manipulator tooth arrangement robot," Robot, 2008, vol. 30, no. 6 , pp. 542-547

[10] Mousavi SA, Hashemipour M., Sadeghi M., Petrofsky, Prowse MA .A fuzzy logic control system for the Rotary dental instruments Iranian Journal of Science \& Technology, Transaction B: Engineering ,2010,vol 34 ,no 5 ..
[11] Kargar V, Mousavi S.A., Foroghi MR, Golestane. H. Q ualyative and quatitative analysys and comparision automatic injection of prophylactic paste with the conventional polishing technique.Journal of Isfahan dental school , 2010, vol 10 ,no.2: 120-127.

About Author :

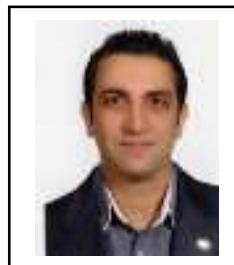

Sayed Ali Mousavi has been a member of ASME since 2003. He received his $\mathrm{PhD}$ in Bio-mechatronics engineering from the Eastern Mediterranean University of Cyprus (EMU), Famagusta, Northern Cyprus, in 2010, his MSc in mechanical engineering from EMU in 2009, and his BSc in mechanical engineering from Isfahan University of Technology Dr. Mousavi was the director of the Robotics Laboratory of Islamic Azad University, Najafabad Branch (IAUN), Najafabad, Isfahan, Iran, in 2011. He has published several articles and papers in books, journal transactions, and conference proceedings. He registered different patents under his name. His research interests are in the areas of biomechanical engineering, mechatronics, and intelligent systems 